MINI-SYMPOSIUM

\title{
Childhood origins of endothelial dysfunction
}

\author{
J P J Halcox, J E Deanfield
}

Heart 2005;91:1272-1274. doi: 10.1136/hrt.2005.061317

A therosclerosis is a chronic vascular inflammatory disease, characterised by lipid accumulation, and cellular infiltration and proliferation in the arterial wall. Vascular endothelial dysfunction plays a critical role in the initiation and progression of the atherosclerotic process; the development of techniques that allow detection of endothelial function and early structural vascular disease has allowed major advances in understanding the pathophysiology at all stages of the disease process. Clinical investigation of endothelial function in children and young adults is informative. Postmortem studies demonstrating the presence of atherosclerotic lesions from as early as the first decade of life have shown that disease burden is directly associated with the extent of exposure to conventional risk factors, even at this early stage. ${ }^{1}$ Longitudinal studies have also demonstrated that this interaction between risk factors and abnormal arterial biology predicts adverse long term cardiovascular outcome. Furthermore, risk factors tend to cluster together and persist from childhood into later life, suggesting potential for early lifestyle and, where appropriate, pharmaceutical intervention. Study of clinical endothelial function in young subjects has confirmed an adverse early impact of both conventional and novel factors. Genetic influences can also be evaluated at this environmentally "naïve" stage of life. Understanding the determinants of early disease progression, based on objective measures of vascular phenotype, provides an opportunity for effective, evidence based preclinical intervention.

\section{ENDOTHELIAL FUNCTION TESTING}

By virtue of its location, the endothelium is optimally located to act as a signal transducer to modulate the impact of circulating influences on the vascular wall. Endothelium derived molecules regulate vasomotor tone, cell adhesion, coagulation, inflammation, and permeability, all of which are central to the atherogenic process. Many circulating endothelium derived adhesion molecules (for example, VCAM-1, ICAM-1, and E-selectin), coagulation factors (for example, von Willebrand factor, tissue plasminogen activator, and plasminogen activator inhibitor-1), and vasoactive mediators (for example, endothelin, prostanoids, and nitric oxide (NO) derivatives) can be measured. While these are potentially useful biomarkers, the majority of clinical studies have focused on endothelial NO-mediated regulation of vasomotor tone since bioavailability of this molecule is central to healthy endothelial function. This can be accurately and reproducibly measured by determination of the vasodilator response to pharmacologic and physiologic stimulation of the endothelium in vivo, and measures of endothelial vasomotor function are predictive of long term outcome. ${ }^{2-4}$

Following the pioneering work of Furchgott and colleagues, coronary and peripheral vascular endothelial function was initially studied by infusion of acetylcholine into the coronary and peripheral arteries of patients. However, these techniques are invasive, potentially risky, are unsuitable for large clinical studies, and have a limited role in children.
Over a decade ago, our laboratory developed a non-invasive technique using high resolution ultrasound to measure endothelium dependent, flow mediated dilation (FMD) of the brachial artery. FMD is mediated by endothelial NO release in response to hyperaemic shear stress. Measurement of FMD is accurate and reproducible, ${ }^{4}$ a good marker of coronary vascular dysfunction, ${ }^{5}$ and is now the most widely applied test of endothelial function and its response to intervention. It remains a technically demanding technique and challenging to apply in large scale clinical trials or routine practice. Other non-invasive techniques have been developed to study vascular dysfunction, including ultrasound assessment of vascular distensibility, tonometric assessment of pulse wave velocity and waveform analysis, and cutaneous microvascular laser Doppler assessment. Although promising, the pathophysiological basis, reproducibility, relation to risk factor burden, contribution of endothelium derived mediators, and prognostic value of these measures require further clarification.

\section{FACTORS CONTRIBUTING TO ENDOTHELIAL DYSFUNCTION IN CHILDHOOD}

Endothelial function deteriorates with increasing age in otherwise healthy subjects, with the rate of decline differing between men and women in line with observed differences in the emergence of clinical disease. ${ }^{6}$ Expression of atherosclerosis related genes is increased in macrophages from male but not female subjects exposed to androgens ${ }^{7}$; considered together with the protective effects of endogenous oestrogen, this helps explain the male predisposition to earlier atherosclerosis. Puberty may therefore be a critical period for the vascular endothelium, but this has not yet been fully characterised.

Endothelial dysfunction is present in children and young adults exposed to conventional risk factors including hypercholesterolaemia, a family history of hypertension, and active and passive smoking. Indeed, conventional risk factors "cluster" in certain individuals, and clear relations emerge between the risk factor "burden" and the degree of endothelial dysfunction. ${ }^{8}$ This has particular relevance for the rapidly expanding population of overweight and obese children with mild hypertension, dyslipidaemia, insulin resistance, and low grade inflammation. Indeed, obese children have impaired FMD and increased vascular stiffness, ${ }^{9}$ which may in part be mediated by leptin.

The role of inflammation in driving the atherogenic process has been shown in numerous large adult cohorts. A link between inflammation, endothelial dysfunction, and structural arterial disease emerges remarkably early. A small study of 79 "healthy" 10 year old children demonstrated an association between mild elevation of $\mathrm{C}$ reactive protein and impaired FMD as well as carotid intimal thickening. ${ }^{10}$ Proinflammatory influences commonly operating in childhood include obesity and exposure to infectious pathogens. We

Abbreviations: ALSPAC, Avon longitudinal study of parents and children; EPC, endothelial progenitor cell; FMD, flow mediated dilation; NO, nitric oxide 
found that mild childhood respiratory infection is associated with, at least partially reversible, endothelial dysfunction, consistent with experimental data describing accelerated atherosclerosis after specific infections and associations between infectious pathogen burden and inflammation, endothelial dysfunction, and clinical coronary artery disease. ${ }^{11}$ Thus, low grade chronic infection may play a more significant role in the initiation and progression of early, preclinical disease than in the destabilisation of advanced disease.

There has been considerable debate about the potential for prenatal "programming" of long term cardiovascular risk. We and others have demonstrated a relation between birthweight, a measure of fetal well being, and endothelial function by the end of the first decade of life. Recent provocative data from our institution suggest that the critical window for metabolic and vascular programming occurs during the very early postnatal period, ${ }^{12}$ suggesting great potential for long term cardiovascular benefits from appropriate early postnatal nutritional modification.

The strong familial and genetic contribution to atherogenesis has long been recognised. Characterisation of early functional and structural vascular disease facilitates evaluation of the genetic contribution by minimising the confounding impact of long term exposure to the complex risk factor burden characteristic of older western populations. We examined the relation between the Glu298Asp polymorphism of the endothelial nitric oxide synthase gene and FMD and found that although there was no relation between the polymorphism and endothelial function in the whole cohort, the 298Asp allele appeared to predispose to endothelial dysfunction in smokers. ${ }^{13}$ This effect was attenuated by increased $\omega-3$ fatty acid concentrations, indicating the potential for genetic variation and specific environmental factors to influence the vascular phenotype from an early stage. Large cohorts will be required to answer questions addressing the potential interactions between specific genes and environmental modifiers with sufficient statistical power. We have recently completed measurements of FMD and vascular stiffness in a well characterised cohort of 8000 10 year old children who were recruited prospectively and followed up from pregnancy in the Avon longitudinal study of parents and children (ALSPAC). This study will provide a unique opportunity to explore in detail the impact of genetic and environmental factors and their interactions at this early stage of life, and examine how these factors drive the atherosclerotic process through puberty and into adult life.

\section{RESTORATION AND PROTECTION OF ENDOTHELIAL FUNCTION REVERSIBILITY}

Endothelial dysfunction is directly relevant to the risk and progression of atherosclerosis. Pharmaceutical intervention with statins and angiotensin converting enzyme (ACE) inhibitors can improve endothelial function and modify progression to clinical disease. However, such measures are currently only considered appropriate for young subjects at very high risk. Several studies have now demonstrated improvement in endothelial function with exercise training and nutritional supplementation with L-arginine, niacin, and n-3 fatty acids. This is particularly exciting for young, at-risk subjects in whom lifestyle modification is the first line approach to risk reduction. Furthermore, as the disease process is most likely to be reversible at this stage, aggressive lifestyle intervention will maximise the cumulative "investment" benefit.

The recent identification of a circulating population of bone marrow derived endothelial progenitor cells (EPCs) has stimulated considerable research interest, particularly into their role in angiogenesis and myocardial repair in patients with myocardial ischaemia and infarction. EPCs may also serve as an endogenous vascular repair mechanism. Reduced circulating numbers of EPCs are associated with endothelial dysfunction in human subjects at increased risk of atherosclerosis. ${ }^{14}$ However, FMD is preserved in at-risk individuals with higher numbers of circulating EPCs, suggesting a protective role against vascular injury and development of atherosclerosis. This concept has been explored further in a mouse model, in which the transfusion of bone marrow cells from younger donors attenuates atherogenesis and inflammation, suggesting a link between these processes and aging that could be modulated by bone marrow derived cells. ${ }^{15} 16$

The management of subjects both with and at risk of clinical atherosclerosis has been improved dramatically over recent decades by treatments such as statins and stents. However, the population burden of atherosclerotic disease may be increasing as a consequence of the deteriorating risk profile of the young population. We now have non-invasive techniques that are accurate, reproducible, reflect the biology of the vascular wall, link to long term prognosis, and respond to appropriate interventions which can be used to quantify progression of vascular disease in early life. These measures are ideal for use as surrogate end points in clinical studies, since the low clinical morbidity and mortality from this disease in the young precludes the use of clinical events as a primary outcome measure. This provides us with a valuable opportunity to study the early evolution of atherosclerosis and should assist in the design of effective risk reduction strategies in the young.

\section{Authors' affiliations}

J P J Halcox, J E Deanfield, Vascular Physiology Unit, Department of Cardiology, Institute of Child Health, University College London, London, UK

Dr Halcox and Professor Deanfield are supported by the British Heart Foundation

Neither author has any relevant conflict of interest to declare

Correspondence to: Dr Julian P J Halcox, Vascular Physiology Unit, Institute of Child Health, 30 Guildford Street, London WCIN 1EH, UK; j.halcox@ich.ucl.ac.uk

\section{REFERENCES}

1 Berenson GS, Srinivasan SR, Bao W, et al. Association between multiple cardiovascular risk factors and atherosclerosis in children and young adults. The Bogalusa heart study. N Engl J Med 1998;338:1650-6.

2 Halcox JP, Schenke WH, Zalos G, et al. Prognostic value of coronary vascular endothelial dysfunction. Circulation 2002;106:653-8.

3 Mullen MJ, Kharbanda RK, Cross J, et al. Heterogenous nature of flowmediated dilatation in human conduit arteries in vivo: relevance to endothelial dysfunction in hypercholesterolemia. Circ Res $2001 ; 88: 145-51$.

4 Sorensen KE, Celermajer DS, Spiegelhalter DJ, et al. Non-invasive measurement of human endothelium dependent arterial responses: accuracy and reproducibility. Br Heart J 1995;74:247-53.

5 Anderson TJ, Uehata A, Gerhard MD, et al. Close relation of endothelial function in the human coronary and peripheral circulations. J Am Coll Cardiol 1995;26:1235-41.

6 Celermajer DS, Sorensen KE, Spiegelhalter DJ, et al. Aging is associated with endothelial dysfunction in healthy men years before the age-related decline in women. J Am Coll Cardiol 1994;24:471-6.

7 Ng MK, Quinn CM, McCrohon JA, et al. Androgens up-regulate atherosclerosis-related genes in macrophages from males but not females: molecular insights into gender differences in atherosclerosis. J Am Coll Cardiol 2003;42:1306-13.

8 Celermajer DS, Sorensen KE, Bull C, et al. Endothelium-dependent dilation in the systemic arteries of asymptomatic subjects relates to coronary risk factors and their interaction. J Am Coll Cardiol 1994;24:1468-74.

9 Tounian P, Aggoun Y, Dubern B, et al. Presence of increased stiffness of the common carotid artery and endothelial dysfunction in severely obese children: a prospective study. Lancet $2001 ; 358: 1400-4$.

10 Jarvisalo MJ, Harmoinen A, Hakanen $M$, et al. Elevated serum $C$-reactive protein levels and early arterial changes in healthy children. Arterioscler Thromb Vasc Biol 2002;22:1323-8.

11 Prasad A, Zhu J, Halcox JP, et al. Predisposition to atherosclerosis by infections: role of endothelial dysfunction. Circulation 2002;106:184-90.

12 Singhal A, Fewtrell M, Cole TJ, et al. Low nutrient intake and early growth for later insulin resistance in adolescents born preterm. Lancet 2003;361: 1089-97. 
13 Leeson CP. Hingorani AD, Mullen MJ, et al. Glu298Asp endothelial nitric oxide synthase gene polymorphism interacts with environmental and dietary factors to influence endothelial function. Circ Res 2002;90:1153-8.

14 Hill JM, Zalos G, Halcox JP, et al. Circulating endothelial progenitor cells, vascular function, and cardiovascular risk. N Engl J Med 2003;348:593-600.
15 Edelberg JM, Tang L, Hattori K, et al. Young adult bone marrow-derived endothelial precursor cells restore aging-impaired cardiac angiogenic function. Circ Res 2002;90:E89-93.

16 Rauscher FM, Goldschmidt-Clermont PJ, Davis BH, et al. Aging, progenitor cell exhaustion, and atherosclerosis. Circulation 2003;108:457-63.

\section{IMAGES IN CARDIOLOGY}

\section{Left ventricular non-compaction diagnosed by real time three dimensional echocardiography}

A 65 year old man was admitted with syncope. An ECG in the emergency department revealed ventricular tachycardia with haemodynamic compromise necessitating electrical cardioversion. Serum electrolytes and post-cardioversion ECG were normal. Two dimensional transthoracic echocardiography showed moderate concentric left ventricular hypertrophy with normal systolic function (panels A, B). Cardiac catheterisation showed no evidence of obstructive coronary artery disease. The left ventriculogram was peculiar with a double wall appearance to the left ventricular free wall (panel C). A cardiac biopsy was performed at the same time, which showed normal histology. Having identified the abnormal left ventriculogram, the patient underwent real time three dimensional echocardiography (RT3DE) to study the left ventricle in detail (panels D, E). This revealed severe trabeculation of the left ventricular wall, particularly in the
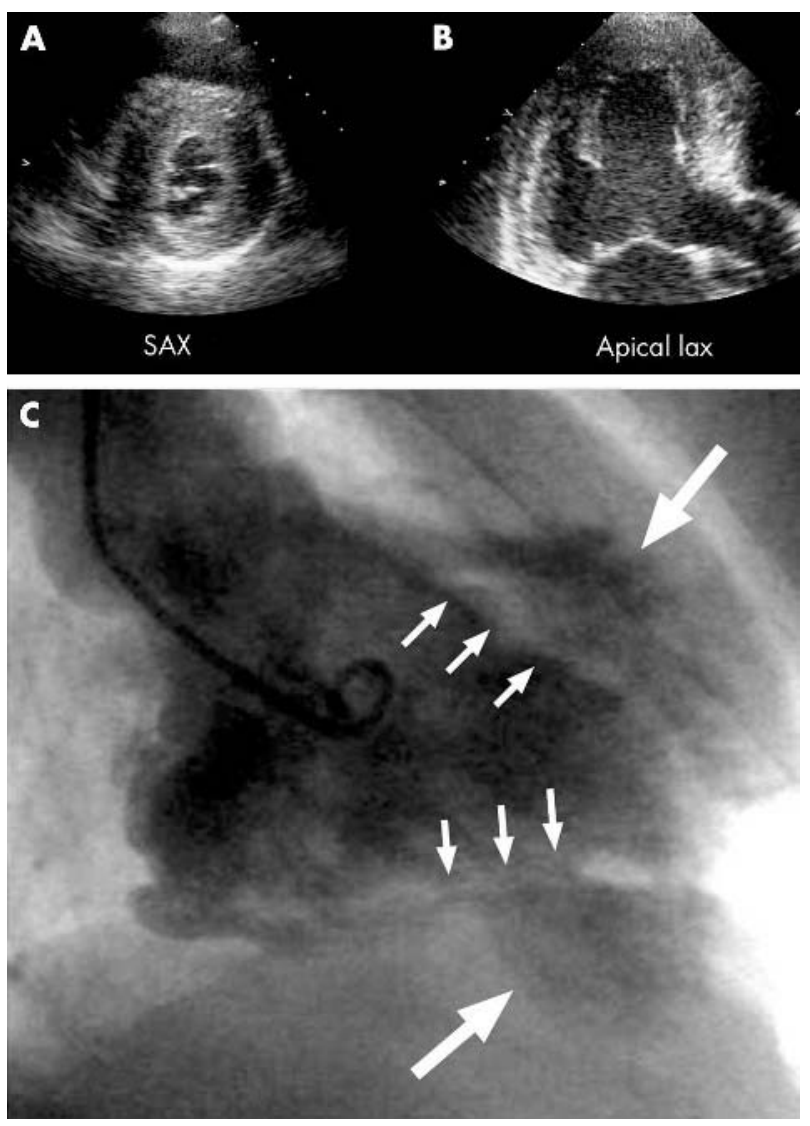

Panels A, B: short axis (SAX) and apical long axis (apical LAX) views on two dimensional echocardiography shows concentric left ventricular hypertrophy only, with normal systolic function. Panel C: left ventriculogram demonstrates a double wall appearance of left ventricular non-compaction. The large and small arrows indicate the outer and inner walls, respectively. apical-lateral segments. Contrast enhanced RT3DE (panels F, $\mathrm{G})$ demonstrated large amounts of filling defects, consistent with extensive trabeculation in the same area. These findings were consistent with non-compaction of the left ventricle (NCLV). The patient was commenced on a $\beta$ blocker and an angiotensin converting enzyme inhibitor, and a cardiac defibrillator was implanted.

This is the first case to report the value of three dimensional echocardiography in the diagnosis of NCLV, an unusual cause of malignant ventricular tachycardia that may be underdiagnosed on two dimensional echocardiography. Moreover, RT3DE allows assessment of any left ventricular dyssynchrony causing heart failure, a recognised complication of NCLV, which helps in planning treatment such as biventricular pacing. These make RT3DE a single superior investigation modality for both the diagnosis and assessment of treatment of LVNC.

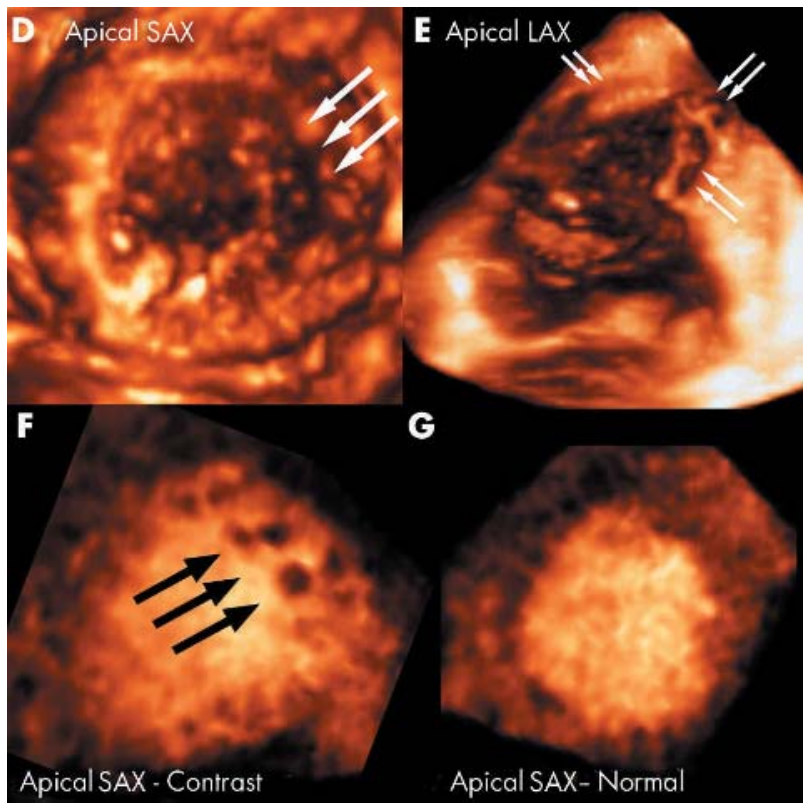

Panels D, E: apical short axis and long axis views on three dimensional echocardiography demonstrate apical hypertrabeculation, consistent with left ventricular non-compaction. The arrows indicate the border between the compacted and non-compacted layers of the myocardium. Panel F: an apical short axis view of the left ventricle from three dimensional contrast echocardiography. The arrows indicate the hypertrabeculations, which project into the cavity. The contrast is seen to fill the intertrabecular recesses. Panel G: a normal short axis view with three dimensional contrast echocardiography for comparison. 